\title{
DESENVOLVIMENTO INICIAL DE MUDAS DE JATOBAZEIRO DO CERRADO EM AQUIDAUANA-MS ${ }^{1}$

\author{
EDILSON COSTA², PAULO ADEMAR MARTINS LEAL ${ }^{3}$, \\ NORTON HAYD REGO ${ }^{2}$, JARBAS BENATTI ${ }^{4}$
}

RESUMO- A busca por uma alimentação saudável tem aumentado o consumo de frutas e hortaliças, proporcionando maior aceitação das fruteiras do cerrado no mercado. Visando à obtenção de mudas de qualidade, este trabalho avaliou a formação de mudas do jatobazeiro-do-cerrado em diferentes ambientes protegidos e substratos, na UEMS, Aquidauana, no período de novembro de 2008 a março de 2009. Foram utilizados três ambientes protegidos: estufa plástica de polietileno transparente, viveiro de tela de sombreamento e viveiro telado com malha termorrefletora; e seis substratos: solo, Plantmax ${ }^{\circledR}$, fibra de coco fina, fibra de coco chips, vermiculita e composto orgânico. O delineamento experimental foi o inteiramente casualizado, com esquema de parcelas subdivididas (split-plot) e dez repetições. Houve interação entre ambiente de cultivo e substrato na formação de mudas de jatobá-do-cerrado. Na estufa agrícola, indica-se a utilização do Plantmax ${ }^{\circledR}$ e, nos telados, recomenda-se a vermiculita. O Plantimax ${ }^{\circledR}$ promoveu crescimento uniforme das mudas, em todos ambientes, especialmente na estufa agrícola. O substrato com $100 \%$ de composto orgânico não é indicado na formação de mudas de jatobá-do-cerrado. O Índice de Qualidade de Dickson é um bom indicador do padrão de qualidade das mudas.

Termos para indexação: Himenaea stigonocarpa, frutas do cerrado, substratos, ambiente de cultivo protegido.

\section{INITIAL SEEDLING DEVELOPMENT OF “JATOBAZEIRO DO CERRADO" IN AQUIDAUANA-MS}

\begin{abstract}
Nowadays, the consumption of fruits and vegetables increased, mainly fruit trees from Cerrado. This work aimed to study the growth of "jatobazeiro do cerrado" seedlings under protected cultivation using different substrates, at the UEMS, Aquidauana, from November 2008 to March 2009. Three protected environments were used: greenhouse with transparent low density polyethylene plastic; black shading screen nursery and thermal reflector screen nursery; and six substrates: soil, Plantmax ${ }^{\circledR}$, fine coconut fiber, chips coconut fiber, vermiculite and organic compound. The experiment was analyzed in a completely randomized design using a split-plot scheme with ten replications. There was interaction between environment and substrate on the growth of "jatobazeiro do cerrado" seedlings. At the greenhouse is indicated the use of the Plantmax ${ }^{\circledR}$ and at the nurseries screen is recommended the vermiculite. The Plantmax ${ }^{\circledR}$ promoted uniform seedling growth in all environments, especially in greenhouse. The substrate with $100 \%$ organic compound is not indicated for the formation of "jatobazeiro do cerrado" seedling. The Dickson Quality Index is a good standard pattern for seedling quality.
\end{abstract}

Index terms: Himenaea stigonocarpa, cerrado fruits, substrates, protected cultivation environment.

\footnotetext{
${ }^{1}$ (Trabalho 041-10). Recebido em: 01-02-2010. Aceito para publicação em: 19-11-2010.

${ }^{2}$ Professor Doutor da UEMS, Unidade de Aquidauana. Rodovia Aquidauana - Cera, Km 12, Zona Rural, C. P. 25, CEP 79200-000, Aquidauana-MS, E-mails: mestrine@uems.br; norton@uems.br

${ }^{3}$ Professor Doutor, FEAGRI-UNICAMP, Campinas - SP, E-mail: pamleal@agr.unicamp.br

${ }^{4}$ Graduando da UEMS, Unidade de Aquidauana, E-mail: jarbasbenatti21@hotmail.com
} 


\section{INTRODUÇÃO}

O jatobá-do-cerrado (Hymenaea stigonocarpa Mart. ex Hayne) é uma importante fruteira do cerrado brasileiro, sendo os frutos utilizados como farinha e na preparação de "cookies" e "snacks" (SILVA et al., 1999; CHANG et al., 1998; VIEIRA et al., 2008). Devido à demanda, aumentou-se a procura e há necessidade de implantação de áreas de plantios comerciais. Em plantio puro no cerrado de Hymenaea spp. (jatobá), Sano e Fonseca (2003) observaram que as plantas atingiram altura média de $1,36 \mathrm{~m}$ em nove anos, apresentando crescimento lento.

A formação de mudas do jatobá-do-cerrado é feita a partir de sementes submetidas à escarificação mecânica (lixadas), e o crescimento inicial das plantas é lento. Botelho et al. (2000) encontraram plantas com 18,16 cm aos seis meses de idade; Campos e Uchida (2002) verificaram 31,45 $\mathrm{cm}$ aos 82 dias após a semeadura e $36,35 \mathrm{~cm}$ aos 150 dias. Sano e Fonseca (2003) obtiveram em Hymenaea spp. altura de $33 \mathrm{~cm}$ aos nove meses. Carvalho (2007) relata que a emergência de plântulas tem início de 9 a 60 dias após a semeadura e que, com três meses de idade, as mudas estão prontas para ir a campo, com porte adequado para o plantio.

A produção de mudas em ambientes protegidos promove melhor crescimento e desenvolvimento da planta, resultando em melhores produções a campo (CAVALCANTE et al., 2002). Para Zanella et al. (2006), a utilização de sombreamento é uma importante técnica na formação de mudas de fruteiras, pois afeta diretamente o crescimento da planta e, posteriormente, a formação do pomar. Contudo, Araújo et al. (2006) obtiveram melhores mudas de mamoeiro em ambiente a céu aberto, em comparação com a estufa plástica e viveiro telado, assim como Mendonça et al. (2008) com tamarindeiro e Carvalho Filho et al. (2003) com jatobá obtiveram melhores mudas em ambiente a céu aberto em comparação com o telado a $50 \%$.

Em mudas de angelim (CARVALHO FILHO et al., 2004) e canafístola (CARVALHO FILHO et al., 2002), o telado promoveu melhores características às mudas. Em casa de vegetação climatizada, Mendonça et al. (2005) obtiveram melhores mudas de mamoeiro "Formosa" do que em viveiro telado por Sombrite ${ }^{\circledR}$ a 50\%. Níveis de sombreamento de $0 ; 30$ e $50 \%$ não influenciaram na altura e diâmetro do colo de Eriotheca pubescens (CARRIJO et al., 2009); no entanto, em mudas de turco (Parkinsonia aculeata L.), nível de $0 \%$ de sombreamento promoveu melhores mudas que o telado de $75 \%$, de acordo com Farias Júnior et al. (2007). Em mudas de maracujá, Costa et al. (2009) encontraram que a tela de monofilamento,com 50\% de sombreamento, promoveu plantas maiores que em estufa plástica.

A formação de mudas em ambiente protegido é realizada em recipientes contendo substratos. O substrato tem a função de sustentação e nutrição inicial da planta, assegurando as condições físicoquímicas que favoreçam o desenvolvimento radicular (ANDRIOLO et al., 1997). A variação na composição destes substratos pode resultar em problemas, podendo ocorrer a não germinação das sementes ou o desenvolvimento irregular das plantas (MINAMI, 1995). A disponibilidade do substrato na região de produção diminui custos, além da facilidade de obtenção, porém Pereira et al. (2008) ressaltam a importância de esterilidade biológica do material utilizado como qualidade desejável em um substrato.

Este trabalho teve como objetivo avaliar o efeito de ambientes protegidos e diferentes substratos no crescimento e desenvolvimento de mudas do jatobá-do-cerrado em Aquidauana-MS.

\section{MATERIAL E MÉTODOS}

O experimento sobre o crescimento inicial de jatobazeiro-do-cerrado foi conduzido na Unidade Universitária de Aquidauana, Universidade Estadual de Mato Grosso do Sul, que se localiza a altitude de $174 \mathrm{~m}$, longitude de $55^{\circ} 40^{\prime} \mathrm{W}$ e latitude de $20^{\circ}$ $27^{\prime}$ S, de outubro de 2008 a março de 2009. O clima da região, de acordo com a classificação de Köeppen é Aw, definido como clima tropical úmido e com temperatura média anual de $24,5^{\circ} \mathrm{C}$.

O crescimento inicial do jatobá-do-cerrado foi avaliado em diferentes ambientes de cultivo e substratos. Utilizaram-se três diferentes ambientes protegidos: (A1) estufa agrícola em arco $(6,40 \mathrm{~m} \mathrm{x}$ $18,00 \mathrm{~m} \times 4,00 \mathrm{~m})$ com estrutura de aço galvanizado, com abertura zenital na cumeeira, coberta com filme polietileno de $150 \mu \mathrm{m}$, difusor de luz, possuindo tela termorrefletora de $50 \%$ sob o filme e fechamentos laterais e frontais com tela de monofilamento, malha para 50\% de sombra; (A2) viveiro agrícola telado, com estrutura de aço galvanizado $(6,40 \mathrm{~m} \times 18,00 \mathrm{~m}$ x 3,50), fechamento em $45^{\circ}$ de inclinação, com tela de monofilamento, malha com $50 \%$ de sombra, e (A3) viveiro agrícola telado com estrutura de aço galvanizado $(6,40 \mathrm{~m} \times 18,00 \mathrm{~m} \times 3,50)$, fechamento em $45^{\circ}$ de inclinação, com tela termorrefletora com $50 \%$ de sombra (Aluminet ${ }^{\mathbb{R}}$ ).

Vasos com capacidade de 5,0 L foram preenchidos com substratos comerciais, solo e composto orgânico, sendo: (S1) solo; (S2) Plantmax ${ }^{\circledR}$; (S3) fibra de coco fina; (S4) fibra de coco grossa (chips); (S5) 
vermiculita, e (S6) composto orgânico Organosuper ${ }^{\circledR}$.

Para avaliação do experimento, foi utilizado o delineamento inteiramente casualizado, em esquema de parcelas subdivididas (03 ambientes x 06 substratos), com dez repetições (plantas), sendo as parcelas principais os ambientes de cultivo, e as subparcelas, os substratos.

As sementes de jatobá-do-cerrado foram coletadas de árvores na região de Aquidauana-MS, nos meses de setembro e outubro de 2008. Foram selecionadas sementes com comprimento médio de 19,00 $\pm 2,00 \mathrm{~mm}$ (BOTELHO et al., 2000). Foi retirada a polpa dos frutos e separadas as sementes que foram lavadas em água corrente e imersas em água por 24 horas. Posteriormente, foram secadas à sombra e escarificadas na área do hilo com lixa d'água número 40.

O solo utilizado, classificado como Argissolo Vermelho-Amarelo, foi retirado na camada de 10 a $40 \mathrm{~cm}$ (Tabela 1). Aos substratos S1 a S5 foram adicionados $14 \%$ de composto orgânico Organosuper ${ }^{\circledR}$ $(\mathrm{pH}=6,51$; Carbono orgânico $=26,2 \%$; Umidade $=$ 4,56\%; Nitrogênio $=1,83 \%$; Fósforo $=0,96 \%$; Potássio $=0,35 \%$; Cálcio $=6,24 \%$; Magnésio $=0,88 \%$; Sódio $=0,23 \%$. Fonte: Laboratório da Embrapa Agropecuária Oeste, Dourados-MS), em volume, e doses de 2,5 kg de superfosfato simples $\left(\mathrm{P}_{2} \mathrm{O}_{5}\right), 0,3$ $\mathrm{kg}$ de cloreto de potássio $(\mathrm{KCl})$ e $1,5 \mathrm{~kg}$ de calcário dolomítico "filler" (PRNT de 100\% de pureza), por metro cúbico de substrato (RIBEIRO et al., 1999). Não se aplicou nitrogênio, pois uma fração dos teores estava presente no composto orgânico na forma mineral (amoniacal e nítrica).

Os substratos ficaram em repouso nos vasos por 12 dias antes da realização da semeadura, dentro dos ambientes de cultivo, para melhor mineralização da matéria orgânica presente no composto orgânico, sendo irrigados manualmente. $\mathrm{O}$ procedimento de irrigação, utilizado durante a condução do experimento, foi manter a umidade do substrato próxima à capacidade de campo. $\mathrm{O}$ preparo do substrato e o preenchimento dos recipientes foram realizados em outubro de 2008. A semeadura ocorreu dia 05 de novembro de 2008 , sendo colocadas duas sementes por recipiente. No dia 13 de dezembro 2008, foi realizado o desbaste, quando as plântulas estavam com duas folhas definitivas.

Foram realizadas mensurações de alturas das plantas (AP), a cada 15 dias, iniciando-se 53 dias após a semeadura (DAS). As demais datas de mensuração de altura foram: aos 68; 83; 98; 113 e 120 DAS. Para a variável altura, em função do tempo, foi realizada análise de regressão com software Excel.

Aos 120 DAS, foram mensuradas as massas da matéria fresca da parte aérea (MFA) e do sistema radicular (MFR), sendo posteriormente levadas em estufa para a secagem e a obtenção das massas das matérias secas da parte aérea (MSA) e do sistema radicular (MSR). Somaram-se as MSA e MSR para obtenção de massa da matéria seca total (MST). Avaliou-se, também, o diâmetro do colo (DC) das plantas. Foram determinadas as relações altura, diâmetro do colo $^{-1}$ (RAD), massa da matéria seca aérea, radicular $^{-1}$ (RMS) e índice de qualidade de Dickson (IQD) (DICKSON et al., 1960):

$$
I Q D=\frac{M S T(\mathrm{~g})}{\frac{A P(\mathrm{~cm})}{D C(\mathrm{~mm})}+\frac{M S A(\mathrm{~g})}{M S R(\mathrm{~g})}}
$$

Foram mensuradas diariamente as temperaturas de bulbo seco e bulbo úmido, às 09; 12 e 15h em cada ambiente de cultivo, no período de 10 de dezembro a 29 de janeiro de 2009. Posteriormente, foi determinada a umidade relativa com auxílio do software Psychrometric Function Demo (Tabela 2).

Os dados foram submetidos à análise de variância, pelo teste $\mathrm{F}$, a 5\% de probabilidade, e, quando significativa, as médias foram comparadas pelo teste de Tukey, ao nível de $5 \%$ de probabilidade, utilizando software Estat (1994).

\section{RESULTADOS E DISCUSSÃO}

Aos 120 dias após a semeadura, foi verificado alta porcentagem de sobrevivência das plântulas nos ambientes de cultivo (parcelas); onde, na estufa e no telado de monofilamento, foram encontradas médias de $87,5 \%$ e, no telado de tela termorrefletora, média de $90,3 \%$. Resultados de sobrevivência mostram satisfatória adaptação das mudas de jatobazeiro-docerrado nos ambientes protegidos.

As parcelas (ambientes), subparcelas (substratos) e interações foram significativas para o crescimento de plântulas (AP), em todas as coletas realizadas (Tabela 3 ). Os substratos promoveram plantas com alturas similares no ambiente A3. No ambiente A2, o substrato S6 apresentou as menores mudas na maioria das coletas. Na estufa agrícola (A1), destacou-se o substrato S2 (Tabela 4).

Para o S3, em todas as coletas, assim como o S6 a partir de 83 DAS, para o S5 aos 98 e 113 DAS, para o S2 na maioria das coletas e para a fibra de coco chips (S4) até 83 DAS, os ambientes não diferiram, apresentando plantas com alturas semelhantes. Para o substrato solo (S1), em todas as coletas, para o S4 a partir de 98 DAS e para o S5 até 83 DAS, os ambientes telados (A2 e A3) promoveram as maiores 
plântulas. Para o substrato S6 até 68 DAS, para o S2 aos 83 DAS e para o S5 aos 120 DAS, o ambiente A2 apresentou as menores mudas (Tabela 4).

$\mathrm{O}$ crescimento do jatobazeiro-do-cerrado é lento, passando de 18,2 cm aos 53 DAS para 27,9 aos 120 DAS (média geral dos substratos e ambientes), ou seja, um crescimento médio de $9,7 \mathrm{~cm}$ em 67 dias. $\mathrm{Na}$ estufa, as mudas no Plantmax ${ }^{\circledR}$, com $35,2 \mathrm{~cm}$, foram $13,4 \mathrm{~cm}$ maiores que aquelas em solo $(21,8$ $\mathrm{cm})$ aos 120 DAS. Sano e Fonseca (2003) obtiveram, em jatobá (Hymenaea courbaril L.), altura de $33 \mathrm{~cm}$ aos 270 DAS. Campos e Uchida (2002) verificaram $31,45 \mathrm{~cm}$ aos 82 DAS e $36,35 \mathrm{~cm}$ aos 150 DAS.

Botelho et al. (2000) verificaram que, aos seis meses de idade, as mudas apresentaram em média $18,16 \mathrm{~cm}$ de altura e diâmetro de $5,52 \mathrm{~mm}$ e, no presente trabalho, aos $120 \mathrm{DAS}$, as plantas atingiram altura média geral de $27,85 \mathrm{~cm}$ e diâmetro médio geral de $5,43 \mathrm{~cm}$.

$\mathrm{Na}$ estufa agrícola, por apresentar maior evapotranspiração (ARAUJO et al., 2006), foi necessário maior número de regas, assim como maior quantidade de água, em alguns dias, no decorrer do experimento. Essa maior perda de água foi provocada pelo efeito estufa do filme de polietileno (CUNHA; ESCOBEDO, 2003), o qual diminui a perda de calor do ambiente para o meio externo, devido às ondas longas de calor, elevando a temperatura e diminuindo a umidade relativa do ar (COSTA et al., 2004). Nesse ambiente, esse processo exige maior gasto de energia das plântulas nos substratos com maior condutividade hidráulica (S4) ou menor quantidade de poros (S1) (KRAMER; BOYER, 1995), que reduz a disponibilidade de água e diminui o contato com raízes, resultando em mudas menores em relação aos ambientes telados (Tabela 4).

As temperaturas médias nos ambientes de cultivo durante o experimento, nos horários de coletas, foram praticamente iguais, porém a umidade relativa foi menor na estufa plástica, provavelmente pela maior evapotranspiração nesse ambiente (Tabela 2). Costa et al. (2009) também não observaram diferenças de temperaturas máximas e mínimas em ambientes semelhantes aos estudados neste trabalho, nos mesmos horários de coletas.

As Figuras 1 e 2 apresentam os ambientes (parcelas) e os substratos (subparcelas), independentemente de suas interações, onde foi observado aos 120 DAS, em valores absolutos de crescimento, maiores plantas nos telados (A2 e A3) e no Plantmax ${ }^{\circledR}(\mathrm{S} 2)$.

Todos os tratamentos, envolvendo conjuntamente ambientes e substratos, promoveram plantas acima de 15 centímetros a partir dos 53 DAS, atingindo até $35,2 \mathrm{~cm}$ aos 120 DAS (Tabela 4), diferentemente do obtido por Santos et al. (2007), que encontraram médias de $11,17 \mathrm{~cm}$ aos cinco meses de avaliação (150 DAS), provavelmente por não escarificarem as sementes.

O diâmetro do colo (DC), a massa da matéria fresca da parte aérea (MFA) e a relação altura e diâmetro (RAD) não foram significativos nos ambientes de cultivo, assim como a interação ambiente e substrato (A x S) do diâmetro do colo (Tabela 5). Mesmo não havendo diferenças significativas entre os ambientes e entre as interações ambientes e substratos, para o diâmetro do colo, obtiveram-se o menor valor $(4,61$ $\mathrm{mm})$ no substrato $\mathrm{S} 4$ dentro da estufa e o maior valor $(6,43 \mathrm{~mm})$ no substrato $\mathrm{S} 6$ dentro do viveiro com tela termorrefletora, muito superiores aos 1,80 $\mathrm{mm}$ obtido por Santos et al. (2007).

No ambiente A1, o substrato S2 foi similar ao S3 nas biomassas foliares e superior aos demais, os substratos S1 e S6 apresentaram as menores biomassas radiculares e relação altura/diâmetro ${ }^{-1}$, e o S4 a menor relação massa da matéria seca aérea e radicular (RMS). No ambiente A2, o substrato S3 promoveu as menores biomassas da parte aérea e RMS, o S6 as menores radiculares e o S5 a menor RAD. No ambiente A3, os substratos S3 e S4 apresentaram as menores biomassas da parte aérea e RMS, o S6 as menores radiculares e RAD (Tabela 6).

Possivelmente, a má compostagem da fibra de coco fina (S3), aliada aos ambientes que recebiam água pluvial (A2 e A3) proporcionaram menor acúmulo de biomassa aérea (MSA), provavelmente devido ao encharcamento dos vasos nos períodos chuvosos (Tabela 6). No ambiente A1, por possuir o filme de polietileno e não permitir a entrada de água pluvial, houve melhor manejo da irrigação, propiciando às mudas no substrato com fibra de coco maior índice de qualidade de Dickson.

Menor acúmulo de biomassa seca radicular (MSR) foi verificado no composto orgânico (S6), o qual, por apresentar elevada percentagem de matéria orgânica, possuía alto poder de adsorção de água (SILVA et al., 2006; ARAÚJO NETO et al., 2010) e desta forma obstruindo os poros por maior tempo, prejudicando o desenvolvimento radicular (Tabela 6). Além desse aspecto, o composto orgânico no início do experimento (novembro de 2008) poderia estar necessitando de estabilidade biológica e utilizando o nitrogênio para a decomposição da matéria orgânica, e por não ter recebido adubação química antes da semeadura, como os demais substratos, no final do experimento (março de 2009), pode ter sofrido pela escassez de nutrientes, resultando em menor acúmulo 
de biomassa radicular.

Para as biomassas dos substratos S2 e S6, RMS do S6, biomassas radiculares frescas e RAD dos substratos $\mathrm{S} 3$ e $\mathrm{S} 4$, os ambientes não diferiram. Para as biomassas do S1, biomassas radiculares do $\mathrm{S} 5$ e biomassa radicular seca do S4, os viveiros telados foram semelhantes. Para as biomassas aéreas e MSR do S3, para a MFA, RAD e RMS do S5, o telado de monofilamento apresentou os menores valores, contudo apresentou maiores valores para as biomassas do S4 e RAD do S1. Para a MSA do S5 e RMS do S2, o telado aluminizado apresentou maiores valores, assim como a estufa revelou maior RAD do S2 e maior RMS do S3 (Tabela 6). Não houve características marcantes que diferenciassem os ambientes de cultivo nas varáveis de biomassa, RAD e RMS. Esses resultados revelam que todos os ambientes de cultivo foram propícios à formação de mudas de jatobá-do-cerrado.

As menores massas da matéria seca total (MST) foram obtidas no substrato com 100\% de composto orgânico. Possivelmente, a adsorção de água pela matéria orgânica, promovendo menor drenagem, associada a uma possível escassez nutricional, tenha promovido menor acúmulo de carboidratos, minerais, proteínas, fibras e lipídeos da matéria seca nas plantas.

Pelo índice de qualidade de Dickson, a vermiculita (S5) promoveu as mudas mais vigorosas nos ambientes telados e a fibra de coco fina (S3) na estufa agrícola. Para os substratos S1, S2, S4 e S6, os ambientes de cultivo não apresentaram diferenças no IQD das plântulas, no entanto para o S3 os maiores valores foram verificados nos ambientes $\mathrm{A} 1$ e A3, e para o $\mathrm{S} 5$ as mudas do ambiente $\mathrm{A} 3$ apresentaram maior IQD que as do A1 (Tabela 6).

As curvas de crescimento das plantas (Figuras 3; 4 e 5) indicam que, nos viveiros telados (A2 e A3), o menor coeficiente de determinação foi obtido no substrato com vermiculita (S5), apresentando menor uniformidade de crescimento ao longo do tempo que os demais substratos, no entanto foi verificado maior vigor (IQD) nesse substrato. Vários trabalhos relatam as propriedades benéficas da porosidade da vermiculita para a formação de mudas de fruteiras, pois apresenta grande capacidade de aeração e absorção de água (MINAMI,1995; NEGREIROS et al., 2003; COSTA et al., 2009).

A maioria dos substratos dentro dos três ambientes apresentou crescimento uniforme durante o período de experimento (Figuras 3; 4 e 5), com coeficientes de determinação elevados, apresentando incremento linear na altura da muda. O substrato Plantmax $^{\circledR}$ (S2) foi o que apresentou maior correlação de crescimento nos ambientes de cultivo, sendo inferior, apenas, ao substrato com fibra de coco fina no viveiro com Sombrite ${ }^{\circledR}$.

TABELA 1 - Análise do solo (S1) utilizado no experimento. Aquidauana - MS.

\begin{tabular}{cccccccccc}
\hline${ }^{(1)} \mathrm{pH}$ & $\begin{array}{c}\text { M.O. } \\
\%\end{array}$ & $\begin{array}{c}{ }^{(2)} \mathrm{P} \\
\mathrm{mg} \mathrm{dm}^{-3}\end{array}$ & ${ }^{(2)} \mathrm{K}$ & $\mathrm{Ca}$ & $\mathrm{Mg}$ & $\begin{array}{c}\mathrm{H}+\mathrm{Al} \\
\mathrm{mmol}_{c} \mathrm{dm}^{-3}\end{array}$ & $\mathrm{SB}$ & $\mathrm{T}$ & $\begin{array}{c}\mathrm{V} \\
\%\end{array}$ \\
\hline 5,4 & 1,4 & 10,5 & 3,5 & 9,0 & 8,0 & 33,0 & 21,0 & 54,0 & 38,9 \\
\hline
\end{tabular}

Fonte: laboratório de análises do solo da agência estadual de defesa sanitária animal e vegetal de MS.

$\mathrm{MO}=$ matéria orgânica; $\mathrm{P}$ = fósforo; $\mathrm{K}=$ potássio; $\mathrm{Ca}=$ cálcio; $\mathrm{Mg}=$ magnésio; $\mathrm{H}+\mathrm{Al}=$ acidez potencial; $\mathrm{SB}=$ soma de bases;

$\mathrm{T}=$ capacidade de troca de cátions; $\mathrm{V}=$ saturação por bases.

(1) $\mathrm{em} \mathrm{CaCl}_{2}, 1: 2,5$; ${ }^{(2)} \mathrm{P}$ e $\mathrm{H}$ extrator Mehlich 1 .

TABELA 2 - Temperatura $\left({ }^{\circ} \mathrm{C}\right)$ e Umidade relativa (\%) média nos horários das $09 \mathrm{~h}, 12 \mathrm{~h}$ e $15 \mathrm{~h}$ para cada ambiente (A) de cultivo, durante o experimento. Aquidauana - MS.

\begin{tabular}{|c|c|c|c|c|c|c|c|c|c|}
\hline & TBS & TBU & TBS & TBU & TBS & TBU & & UR & \\
\hline & \multicolumn{2}{|c|}{09 Horas } & \multicolumn{2}{|c|}{12 Horas } & \multicolumn{2}{|c|}{15 Horas } & 09 Horas & 12 Horas & 15 Horas \\
\hline A1 & 28,9 & 24,1 & 33,6 & 25,9 & 35,7 & 26,9 & 69,0 & 56,1 & 53,0 \\
\hline $\mathrm{A} 2$ & 28,7 & 24,5 & 33,3 & 26,2 & 35,1 & 27,4 & 72,1 & 58,9 & 57,7 \\
\hline A3 & 29,6 & 24,9 & 33,6 & 26,8 & 35,6 & 27,7 & 70,0 & 60,8 & 57,1 \\
\hline
\end{tabular}

TBS = temperatura de bulbo seco $\left({ }^{\circ} \mathrm{C}\right) ; \mathrm{TBU}=$ temperatura de bulbo úmido $\left({ }^{\circ} \mathrm{C}\right) ; \mathrm{UR}=$ umidade relativa $(\%) ; \mathrm{A} 1=$ Estufa agrícola; $\mathrm{A} 2=$ Viveiro com Sombrite ${ }^{\circledR} ; \mathrm{A} 3=$ viveiro com Aluminet $^{\mathbb{\circledR}}$. 
TABELA 3 - Valores de F calculado da análise de variância (ANOVA) para a altura de planta (AP) do jatobá-do-cerrado avaliada aos 53; 68; 83; 98; 113 e 120 dias após a semeadura (DAS). Aquidauana - MS.

\begin{tabular}{lccccccc}
\hline & & \multicolumn{5}{c}{ DAS } \\
\cline { 3 - 7 } \multicolumn{1}{c}{ CV } & GL & 53 & 68 & 83 & 98 & 113 & 120 \\
\hline A & 2 & $5,05 *$ & $5,87 * *$ & $3,54 *$ & $4,29 *$ & $4,22 *$ & $7,35 * *$ \\
Res. (a) & 27 & & & & & & \\
P & 29 & & & & & & \\
S & 5 & $3,56 * *$ & $5,73 * *$ & $6,29 * *$ & $9,51 * *$ & $11,48 * *$ & $11,98 * *$ \\
A x S & 10 & $1,93 *$ & $2,60 * *$ & $2,28 *$ & $2,72 * *$ & $3,57 * *$ & $3,51 * *$ \\
Res. (b) & 135 & & & & & & \\
Total & 179 & & & & & & \\
\hline
\end{tabular}

$\mathrm{CV}=$ causa de variação; $\mathrm{GL}=$ graus de liberdade; ambientes $=\mathrm{A} ;$ Res $(\mathrm{a})=$ resíduo (a); $\mathrm{P}=$ parcelas; $\mathrm{S}=$ substratos; $\mathrm{A} \times \mathrm{S}=$ interação entre ambiente e substrato; Res (b) = resíduo (b); NS = não significativo; $*$ = significativo $5 \%$; ** = significativo $1 \%$.

TABELA 4 - Alturas de plantas (AP) do jatobá-do-cerrado dos substratos (S) dentro dos ambientes (A) e dos ambientes (A) dentro dos substratos (S). Aquidauana - MS.

\begin{tabular}{|c|c|c|c|c|c|c|}
\hline & A1 & $\mathrm{A} 2$ & A3 & A1 & $\mathrm{A} 2$ & A3 \\
\hline & \multicolumn{3}{|c|}{53 DAS $(\mathrm{cm})$} & \multicolumn{3}{|c|}{68 DAS $(\mathrm{cm})$} \\
\hline $\mathrm{S} 1$ & $15,4 \mathrm{Bb}$ & $18,9 \mathrm{Aa}$ & 18,3 Aab & $17,4 \mathrm{Bb}$ & $21,9 \mathrm{Aa}$ & $20,0 \mathrm{Aab}$ \\
\hline S2 & $20,9 \mathrm{Aa}$ & $18,5 \mathrm{Aa}$ & $20,0 \mathrm{Aa}$ & $24,3 \mathrm{Aa}$ & $21,3 \mathrm{Aa}$ & $22,2 \mathrm{Aa}$ \\
\hline S3 & $17,6 \mathrm{ABa}$ & $18,7 \mathrm{Aa}$ & $17,9 \mathrm{Aa}$ & $18,9 \mathrm{Ba}$ & $20,4 \mathrm{Aa}$ & $20,1 \mathrm{Aa}$ \\
\hline S4 & $17,1 \mathrm{ABa}$ & $19,4 \mathrm{Aa}$ & $19,2 \mathrm{Aa}$ & $19,6 \mathrm{ABa}$ & $22,5 \mathrm{Aa}$ & $22,5 \mathrm{Aa}$ \\
\hline S5 & $16,9 \mathrm{ABb}$ & $18,7 \mathrm{Aab}$ & $20,6 \mathrm{Aa}$ & $18,3 \mathrm{Bb}$ & 21,1 Aab & $23,1 \mathrm{Aa}$ \\
\hline S6 & $16,7 \mathrm{ABab}$ & $14,1 \mathrm{Bb}$ & $18,4 \mathrm{Aa}$ & $18,3 \quad \mathrm{Bab}$ & $15,0 \mathrm{Bb}$ & $20,3 \mathrm{Aa}$ \\
\hline \multicolumn{4}{|c|}{83 DAS $(\mathrm{cm})$} & \multicolumn{3}{|c|}{98 DAS $(\mathrm{cm})$} \\
\hline $\mathrm{S} 1$ & $19,1 \mathrm{Bb}$ & $24,7 \mathrm{Aa}$ & $23,6 \mathrm{Aa}$ & $20,5 \mathrm{Bb}$ & $28,7 \mathrm{Aa}$ & $26,3 \mathrm{Aa}$ \\
\hline $\mathrm{S} 2$ & $28,8 \mathrm{Aa}$ & $24,1 \mathrm{ABb}$ & $25,3 \mathrm{Aab}$ & $31,1 \mathrm{Aa}$ & $28,4 \mathrm{ABa}$ & $29,6 \mathrm{Aa}$ \\
\hline S3 & $21,5 \mathrm{Ba}$ & $21,6 \mathrm{ABa}$ & $22,4 \mathrm{Aa}$ & $24,3 \mathrm{Ba}$ & $23,4 \mathrm{ABCa}$ & $24,1 \mathrm{Aa}$ \\
\hline $\mathrm{S} 4$ & $22,2 \mathrm{Ba}$ & $25,8 \mathrm{Aa}$ & $25,0 \mathrm{Aa}$ & $23,8 \mathrm{Bb}$ & $28,8 \mathrm{Aa}$ & $26,8 \mathrm{Aab}$ \\
\hline S5 & $20,1 \mathrm{Bb}$ & $22,2 \mathrm{ABab}$ & $247 \mathrm{Aa}$ & $22,8 \mathrm{Ba}$ & $22,8 \quad \mathrm{BCa}$ & 27,4 Аa \\
\hline S6 & $20,5 \mathrm{Ba}$ & $18,9 \quad \mathrm{Ba}$ & $22,1 \mathrm{Aa}$ & $22,0 \quad \mathrm{Ba}$ & $21,2 \mathrm{Ca}$ & $24,0 \mathrm{Aa}$ \\
\hline \multicolumn{4}{|c|}{113 DAS $(\mathrm{cm})$} & \multicolumn{3}{|c|}{120 DAS $(\mathrm{cm})$} \\
\hline $\mathrm{S} 1$ & $21,5 \mathrm{Bb}$ & $31,8 \mathrm{Aa}$ & $29,1 \mathrm{Aa}$ & $21,8 \mathrm{Bb}$ & $32,1 \mathrm{ABa}$ & $28,1 \mathrm{Aa}$ \\
\hline $\mathrm{S} 2$ & $34,9 \mathrm{Aa}$ & $32,2 \mathrm{Aa}$ & $31,5 \mathrm{Aa}$ & $35,2 \mathrm{Aa}$ & $32,8 \mathrm{Aa}$ & $32,0 \mathrm{Aa}$ \\
\hline S3 & $26,1 \quad \mathrm{Ba}$ & $25,2 \mathrm{Ba}$ & $26,7 \mathrm{Aa}$ & $26,2 \mathrm{Ba}$ & $26,3 \quad \mathrm{BCa}$ & $27,4 \mathrm{Aa}$ \\
\hline S4 & $25,3 \mathrm{Bb}$ & $31,9 \mathrm{Aa}$ & $28,5 \mathrm{Aab}$ & $25,5 \mathrm{Bb}$ & $32,5 \mathrm{ABa}$ & $29,2 \mathrm{Aab}$ \\
\hline S5 & $25,8 \mathrm{Ba}$ & $23,8 \mathrm{Ba}$ & $28,9 \mathrm{Aa}$ & $24,9 \mathrm{Bab}$ & $24,1 \quad \mathrm{Cb}$ & $29,7 \mathrm{Aa}$ \\
\hline S6 & $23,5 \quad \mathrm{Ba}$ & $23,4 \quad \mathrm{Ba}$ & $25,3 \mathrm{Aa}$ & $23,8 \mathrm{Ba}$ & $23,9 \quad \mathrm{Ca}$ & $26,0 \mathrm{Aa}$ \\
\hline
\end{tabular}

Letras iguais maiúsculas nas colunas e minúsculas nas linhas não diferem entre si, pelo teste de Tukey, a 5\% de probabilidade; A1 = Estufa agrícola; A2 = Viveiro com Sombrite ${ }^{\circledR} ; \mathrm{A} 3={\text { viveiro com } \text { Aluminet }^{\circledR} ; \mathrm{S} 1=\text { solo; S2 = Plantmax }}^{\circledR} ; \mathrm{S} 3=$ fibra de coco fina; S4 = fibra de coco grossa (chips); S5 = vermiculita e S6 = composto orgânico Organosuper ${ }^{\circledR}$. 
TABELA 5 - Valores de F calculado da análise de variância (ANOVA) para o diâmetro do colo (DC), massa da matéria fresca da parte aérea (MFA), massa da matéria seca da parte aérea (MSA), massa da matéria fresca do sistema radicular (MFR), massa da matéria seca do sistema radicular (MSR), relação altura e diâmetro (RAD), relação massa da matéria seca aérea e radicular (RMS), massa da matéria seca total (MST) e índice de qualidade de Dickson (IQD), do jatobá-do-cerrado aos 120 dias após a semeadura. Aquidauana - MS.

\begin{tabular}{lcccccccccc}
\hline \multicolumn{1}{c}{ CV } & GL & DC & MFA & MSA & MFR & MSR & RAD & RMS & MST & IQD \\
\hline A & 2 & 1,61 NS & $1,11^{\text {NS }}$ & 2,07 NS & $3,71 *$ & $6,51 * *$ & $17,57 * *$ & $8,27 * *$ & 0,77 NS & 0,85 \\
Res. (a) & 27 & & & & & & & & & \\
P & 29 & & & & & & & & & \\
S & 5 & $5,22 * *$ & $10,03 * *$ & $6,21 * *$ & $9,48 * *$ & $12,85 * *$ & $88,18 * *$ & $78,12 * *$ & $72,90 * *$ & $18,65 * *$ \\
Ax S & 10 & 1,42 NS & $5,10 * *$ & $5,30 * *$ & $2,28 *$ & $2,98 * *$ & $14,54 * *$ & $17,41 * *$ & $36,13 * *$ & $13,48 * *$ \\
Res. (b) & 135 & & & & & & & & & \\
Total & 179 & & & & & & & & & \\
\hline
\end{tabular}

$\mathrm{CV}=$ causa de variação; $\mathrm{GL}=$ graus de liberdade; ambientes $=\mathrm{A}$; Res. (a) = resíduo (a); $\mathrm{P}=$ parcelas; $\mathrm{S}=$ substratos; $\mathrm{A} x \mathrm{~S}=$ interação entre ambiente e substrato; Res (b) = resíduo (b); ${ }^{\mathrm{NS}}=$ não significativo; $*$ = significativo 5\%; ** = significativo $1 \%$.

TABELA 6 - Biomassas aéreas e radiculares, relação altura e diâmetro (RAD), relação massa da matéria seca aérea e radicular (RMS), massa da matéria seca total (MST) e índice de qualidade de Dickson (IQD) nas interações ambientes e substratos (A x S), aos 120 DAS. Aquidauana - MS.

\begin{tabular}{|c|c|c|c|c|c|c|}
\hline & A1 & $\mathrm{A} 2$ & A3 & A1 & $\mathrm{A} 2$ & A3 \\
\hline & & MFA (g) & & & $\operatorname{MSA}(\mathrm{g})$ & \\
\hline S1 & $6,5 \mathrm{Bb}$ & $9,5 \mathrm{ABa}$ & $8,7 \mathrm{ABCa}$ & $2,9 \mathrm{Bb}$ & 4,6 $\mathrm{ABa}$ & 3,9 ABCab \\
\hline S2 & 10,9 Аa & $9,5 \mathrm{ABCa}$ & $10,2 \mathrm{Aa}$ & 4,8 Aa & 4,4 $\mathrm{ABa}$ & $4,5 \mathrm{ABa}$ \\
\hline S3 & $8,4 \mathrm{ABa}$ & 5,3 & $6,8 \quad \mathrm{Cab}$ & $4,1 \mathrm{ABa}$ & $2,6 \quad \mathrm{Cb}$ & $3,1 \quad \mathrm{Cab}$ \\
\hline S4 & $6,5 \mathrm{Bb}$ & $10,3 \mathrm{Aa}$ & $6,9 \quad \mathrm{Cb}$ & $3,0 \mathrm{Bb}$ & 4,7 Aa & $3,3 \quad \mathrm{BCb}$ \\
\hline S5 & $7,8 \mathrm{Bab}$ & $7,0 \mathrm{BCDb}$ & $9,7 \mathrm{ABa}$ & $3,4 \mathrm{Bb}$ & $3,3 \mathrm{BCb}$ & 4,6 Аa \\
\hline S6 & $6,8 \mathrm{Ba}$ & 6,7 CDa & $7,3 \quad \mathrm{BCa}$ & $3,2 \mathrm{Ba}$ & $3,3 \quad \mathrm{BCa}$ & $3,6 \mathrm{ABCa}$ \\
\hline & & MFR (g) & & & $\operatorname{MSR}(\mathrm{g})$ & \\
\hline S1 & $6,5 \mathrm{BCb}$ & 7,9 ABab & $8,8 \mathrm{ABa}$ & $3,0 \quad \mathrm{CDb}$ & 4,1 Aa & $4,7 \mathrm{ABa}$ \\
\hline S2 & 9,5 Aa & 8,8 Aa & $7,4 \mathrm{ABa}$ & $4,7 \mathrm{Aa}$ & 4,4 Aa & $3,8 \quad \mathrm{BCa}$ \\
\hline S3 & 9,2 Aa & $7,5 \mathrm{ABa}$ & 9,5 Aa & 4,4 $\mathrm{ABab}$ & $3,7 \mathrm{ABb}$ & 5,1 Aa \\
\hline S4 & $8,5 \mathrm{ABa}$ & $8,5 \mathrm{Aa}$ & 9,8 Aa & $4,1 \mathrm{ABCb}$ & $4,3 \mathrm{Aab}$ & 5,3 Aa \\
\hline S5 & $7,2 \mathrm{ABCb}$ & 8,3 $\mathrm{ABab}$ & 9,8 Аa & $3,4 \quad \mathrm{BCDb}$ & $3,8 \mathrm{ABab}$ & $4,8 \mathrm{ABa}$ \\
\hline S6 & $5,1 \quad \mathrm{Ca}$ & $5,7 \quad \mathrm{Ba}$ & $6,7 \mathrm{Ba}$ & 2,5 & $2,8 \mathrm{Ba}$ & $3,1 \quad \mathrm{Ca}$ \\
\hline \multicolumn{4}{|c|}{$\operatorname{RAD}(\mathrm{cm} / \mathrm{mm})$} & \multicolumn{3}{|c|}{ RMS } \\
\hline S1 & 3,97 & $5,99 \quad \mathrm{Ba}$ & $5,23 \mathrm{BCb}$ & $0,99 \quad \mathrm{Ba}$ & 1,11 $\mathrm{ABa}$ & $0,83 \mathrm{Cb}$ \\
\hline S2 & 6,79 Аa & $6,03 \mathrm{Bb}$ & $6,06 \mathrm{Ab}$ & $1,03 \mathrm{Bb}$ & $1,02 \mathrm{Bb}$ & $1,20 \mathrm{Aa}$ \\
\hline S3 & $4,78 \quad \mathrm{Cb}$ & $5,48 \mathrm{Ba}$ & $5,24 \mathrm{BCab}$ & $0,93 \mathrm{Ba}$ & 0,70 & 0,61 \\
\hline S4 & $5,83 \mathrm{Bb}$ & $6,72 \mathrm{Aa}$ & $5,68 \mathrm{ABb}$ & $0,74 \quad \mathrm{Cb}$ & $1,10 \mathrm{ABa}$ & 0,62 \\
\hline S5 & $4,76 \quad \mathrm{Ca}$ & $3,92 \mathrm{Db}$ & $4,79 \mathrm{Ca}$ & $1,01 \quad \mathrm{Ba}$ & $0,87 \quad \mathrm{Cb}$ & $1,00 \mathrm{Bab}$ \\
\hline S6 & 3,89 & $4,62 \quad \mathrm{Ca}$ & 4,04 & $1,30 \mathrm{Aa}$ & $1,24 \mathrm{Aa}$ & $1,22 \mathrm{Aa}$ \\
\hline & \multicolumn{3}{|c|}{$\operatorname{MST}(\mathrm{g})$} & \multicolumn{3}{|c|}{ IQD } \\
\hline $\mathrm{S} 1$ & $\mathrm{Db}$ & $8,7 \mathrm{Aa}$ & $8,6 \mathrm{Ba}$ & $1,18 \mathrm{Ba}$ & $1,22 \mathrm{BCa}$ & $1,41 \quad \mathrm{Ba}$ \\
\hline S2 & 9,5 Aa & 8,8 Аa & $8,3 \mathrm{Ba}$ & $1,23 \mathrm{Ba}$ & $1,26 \mathrm{Ba}$ & $1,14 \quad \mathrm{Ca}$ \\
\hline S3 & $8,6 \mathrm{Ba}$ & $6,4 \mathrm{Cb}$ & $8,3 \mathrm{Bab}$ & $1,49 \mathrm{Aa}$ & 1,05 & $1,43 \quad \mathrm{Ba}$ \\
\hline S4 & $7,1 \quad \mathrm{Ca}$ & 9,1 Аа & $8,6 \mathrm{Ba}$ & $1,15 \mathrm{Ba}$ & $1,16 \quad \mathrm{BCDa}$ & $1,38 \quad \mathrm{Ba}$ \\
\hline S5 & $6,8 \mathrm{Cb}$ & $7,1 \quad \mathrm{Bb}$ & 9,3 Aa & $1,19 \mathrm{Bb}$ & 1,48 Aab & 1,62 Aa \\
\hline S6 & $5,7 \quad \mathrm{Da}$ & $6,1 \quad \mathrm{Ca}$ & $6,7 \quad \mathrm{Ca}$ & $1,11 \mathrm{Ba}$ & $1,07 \quad \mathrm{CDa}$ & $1,28 \mathrm{BCa}$ \\
\hline
\end{tabular}

Letras iguais maiúsculas nas colunas e minúsculas nas linhas não diferem entre si, pelo teste de Tukey, a 5\% de probabilidade; A1 = Estufa agrícola; A2 = Viveiro com Sombrite ${ }^{\circledR} ; \mathrm{A} 3={\text { viveiro com } \text { Aluminet }^{\mathbb{}} ; \mathrm{S} 1=\text { solo; S2 = Plantmax }}^{\circledR} ; \mathrm{S} 3=$ fibra de coco fina; S4 = fibra de coco grossa (chips); S5 = vermiculita e S6 = composto orgânico Organosuper ${ }^{\circledR}$. 


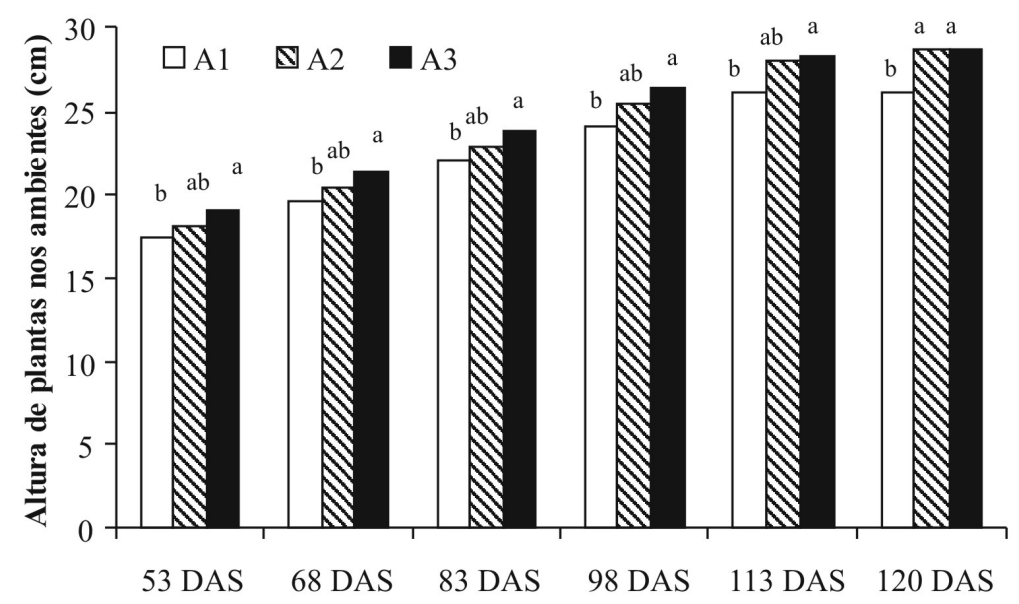

FIGURA 1 - Altura média das mudas de jatobá-do-cerrado nos ambientes de cultivo (A), independentemente do tipo de substrato. Aquidauana - MS.

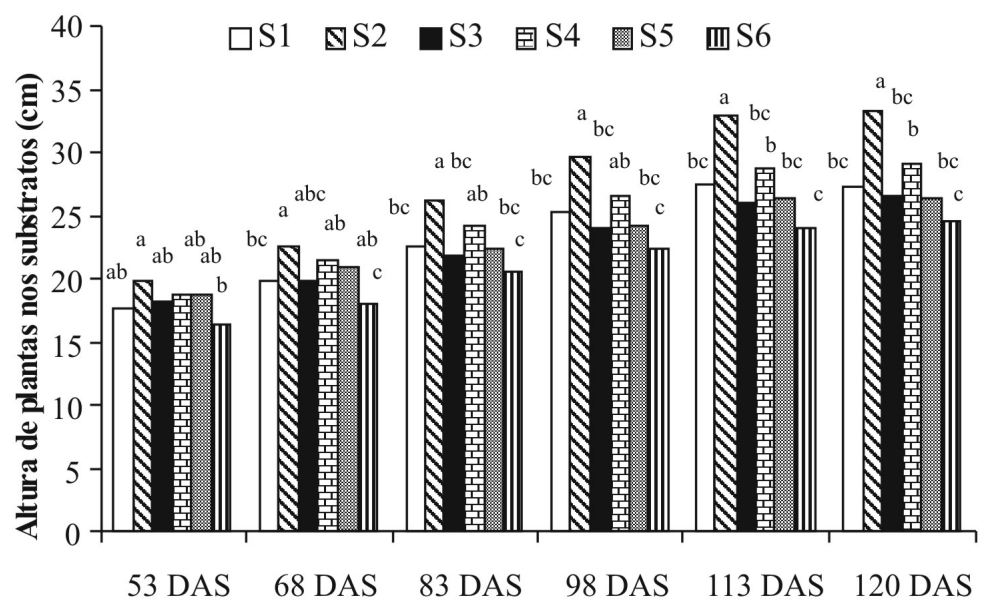

FIGURA 2 - Altura média das mudas de jatobá-do-cerrado nos substratos (S), independentemente do tipo de ambiente de cultivo. Aquidauana - MS. 


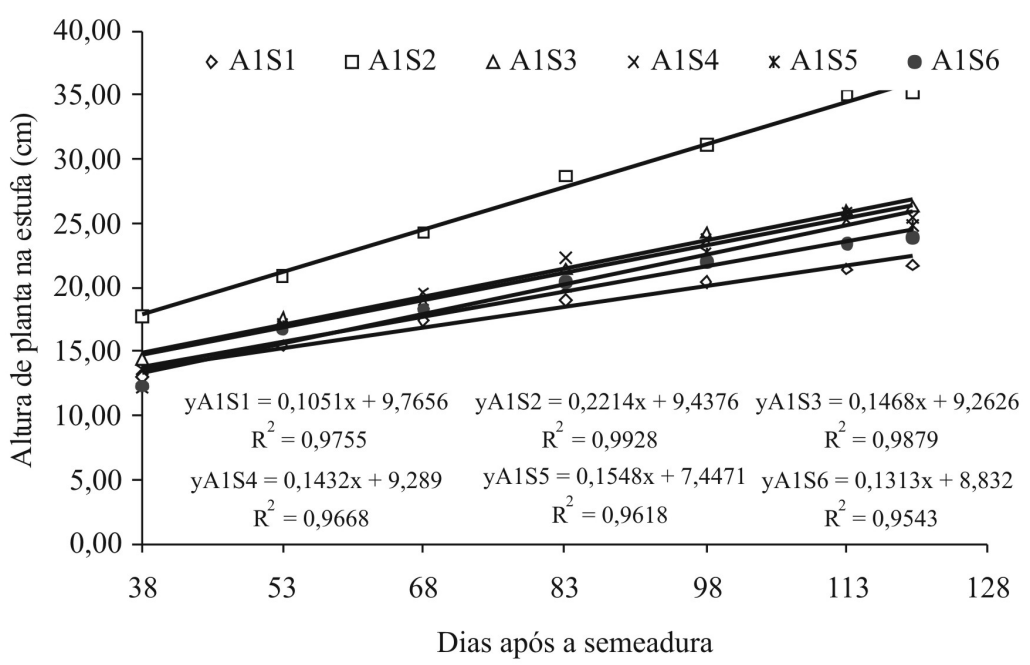

FIGURA 3 -Curva de crescimento das mudas de jatobá-do-cerrado nos diferentes substratos (S), em função do tempo, na estufa agrícola (A1). Aquidauana - MS.

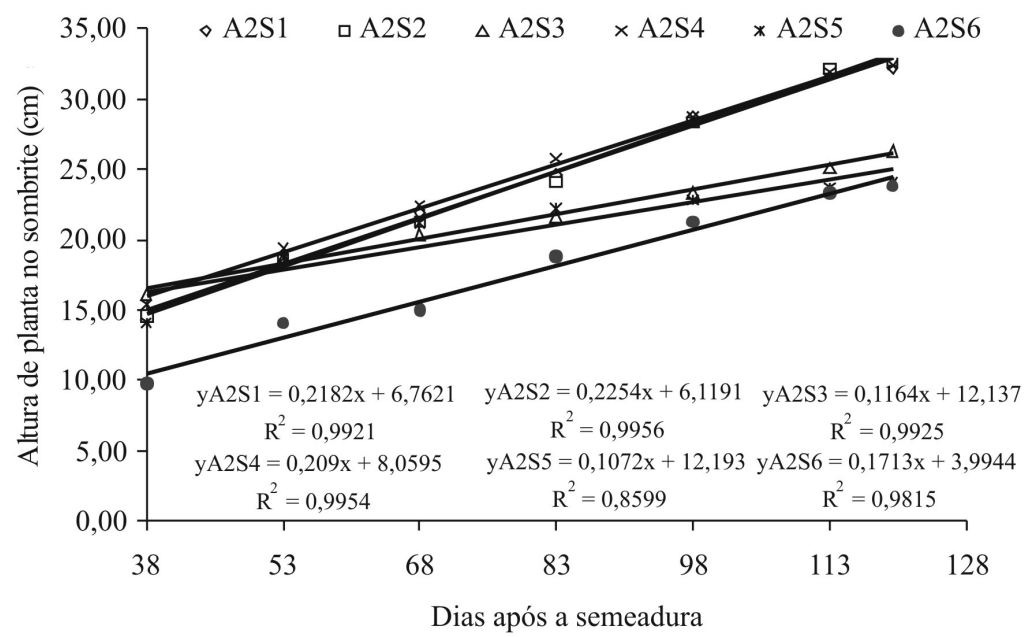

FIGURA 4 -Curva de crescimento das mudas de jatobá-do-cerrado nos diferentes substratos (S), em função do tempo, no viveiro telado com tela de monofilamento de $50 \%$ de sombreamento (A2). Aquidauana - MS. 


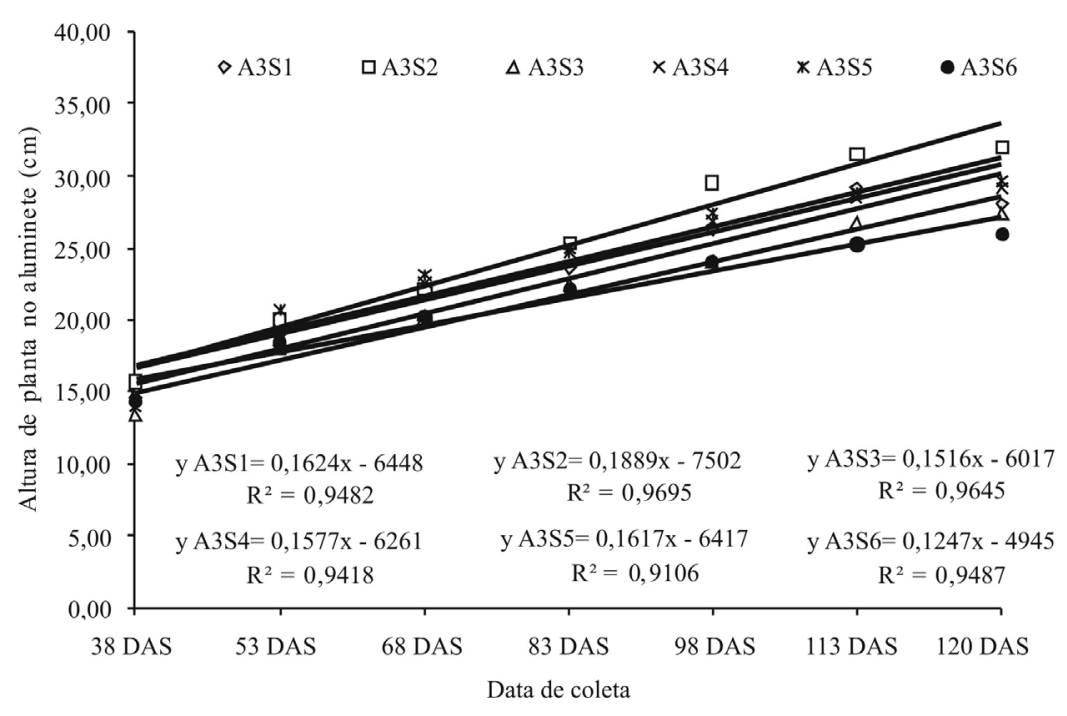

FIGURA 5 - Curva de crescimento das mudas de jatobá-do-cerrado nos diferentes substratos (S), em função do tempo, no viveiro telado com tela termorrefletora de 50\% de sombreamento (A3). Aquidauana - MS.

\section{CONCLUSÕES}

1-Há interação entre ambiente de cultivo e substrato na formação de mudas de jatobá-docerrado. qualidade.

2-Todos os ambientes formam mudas de boa

3-Na estufa agrícola, indica-se a utilização do Plantmax ${ }^{\circledR}$ e, nos telados, recomenda-se a vermiculita.

4-O Plantmax ${ }^{\circledR}$ promove crescimento uniforme em todos ambientes, especialmente na estufa agrícola.

5-O substrato com $100 \%$ de composto orgânico não é indicado à formação de mudas de jatobá-do-cerrado.

6-O Índice de Qualidade de Dickson é um bom indicador do padrão de qualidade das mudas.

\section{AGRADECIMENTOS}

À Pró-Reitoria de Pesquisa e Pós-Graduação (PROPP) da Universidade Estadual de Mato Grosso do Sul (UEMS), pelos recursos de auxílio à pesquisa, tornando possível a realização deste trabalho.

\section{REFERÊNCIAS}

ANDRIOLO, J. L.; DUARTE, T. S.; LUDKE, L.; SKREBSKY, E. C. Crescimento e desenvolvimento do tomateiro cultivado em substrato com fertirrigação. Horticultura Brasileira, Brasília v. 15, n. 1, p. 28-32, 1997.

ARAÚJO, J. G.; ARAÚJO JÚNIOR, M. M.; MENEZES, R. H. N.; MARTINS, M. R.; LEMOS, R. N. S.; CERQUEIRA, M. C. Efeito do recipiente e ambiente de cultivo sobre o desenvolvimento de mudas de mamoeiro cv. sunrise solo. Revista Brasileira de Fruticultura, Jaboticabal, v. 28, n. 3, p. 526-529, 2006.

ARAUJO NETO, S. E.; GALVÃO, R. O.; FERREIRA, R. L. F.; PARMEJIANI, R. S.; NEGREIROS, J. R. S. Plantio direto de cebolinha sobre cobertura vegetal com efeito residual da aplicação de composto orgânico. Ciência Rural, Santa Maria, v. 40, n. 5, p. 1206-1209, 2010. 
BOTELHO, S. A.; FERREIRA, R. A.; MALAVASI, M. M.; DAVIDE, A. C. Aspectos morfológicos de frutos, sementes, plântulas e mudas de jatobá-docerrado (Hymenaea stigonocarpa Mart.ex Hayne) Fabaceae. Revista Brasileira de Sementes, Pelotas, v. 22 , n. 1, p. 144-152, 2000.

CAMPOS, M. A.; UCHIDA, T. Influência do sombreamento no crescimento de mudas de três espécies amazônicas. Pesquisa Agropecuária Brasileira, Brasília, v. 37, n. 3, p. 281-288, 2002.

CARRIJO, C.; MARTINS, R. C. C.; MARTINS, I. S.; LANDAHL, D. T.; MATOS, J. M. de M.; NAKANO, T. Y. R. Estabelecimento de Eriotheca pubescens (Bombacaceae) por meio de semeadura direta e de mudas em cascalheira. CERNE, Lavras, v. 15, n. 3,p.365-370, 2009.

CARVALHO, P. E. R. Jatobá-do-cerrado: Hymenaea stigonocarpa. Colombo: Embrapa Florestas, 2007. 8p. (Circular Técnica, 133)

CARVALHO FILHO, J. L. S. de; ARRIGONIBLANK, M. de F.; BLANK, A. F. Produção de mudas de angelim (Andira fraxinifolia Benth.) em diferentes ambientes, recipientes e composições de substratos. Revista Ciência Agronômica, Fortaleza, v. 35, n. 1, p. 61-67, 2004.

CARVALHO FILHO, J. L. S. de; ARRIGONIBLANK, M. de F.; BLANK, A. F.; SANTOS NETO, A. L. dos; AMANCIO, V. F. Produção de mudas de Cassia grandis L. em diferentes, recipientes e misturas de substratos. Revista Ceres, Viçosa, v. 49, n. 284, p. 341-352, 2002.

CARVALHO FILHO, J. L. S. de; ARRIGONIBLANK, M. de F.; BLANK, A. F.; RANGEL, M. S. A. Produção de mudas de jatobá (Hymenaea courbaril L.) em diferentes ambientes, recipientes e composições de substratos. CERNE, Lavras, v. 9, n. 1, p. 111-121, 2003.

CAVALCANTE, L. F.; SANTOS, J. B.; SANTOS, C. J. O.; FEITOSA FILHO, J. C.; LIMA, E. M.; CAVALCANTE, I. H. L. Germinação de sementes e crescimento inicial de maracujazeiros irrigados com água salina em diferentes volumes de substrato. Revista Brasileira de Fruticultura, Jaboticabal, v. 24, n. 3, p.748-751, 2002.
CHANG, Y. K.; SILVA, M. R.; GUTKOSKI, L.; SEBIO, L.; SILVA, M. A. A. P. Development of extruded snacks using jatobá [Hymenaea stigonocarpa Mart] flour and cassava starch blends. Journal of the Science of Food and Agriculture, London, v. 78, p. 59-66, 1998.

COSTA, E.; LEAL, P. A. M.; CARMO JUNIOR, R. R. Modelo de simulação da temperatura e umidade relativa do ar no interior da estufa plástica. Revista Engenharia Agrícola, Jaboticabal, v. 24, n. 1, p. 57-67, 2004.

COSTA E.; RODRIGUES E. T.; ALVES V. B.; SANTOS, L. C. R.; VIEIRA L. C. R. Efeitos da ambiência, recipientes e substratos no desenvolvimento de mudas de maracujazeiroamarelo em Aquidauana - MS. Revista Brasileira de Fruticultura, Jaboticabal, v. 31, n. 1, p. 236-244, 2009.

CUNHA, A. R.; ESCOBEDO, J. F. Alterações micrometeorológicas causadas pela estufa plástica e seus efeitos no crescimento e produção da cultura de pimentão. Revista Brasileira de Agrometeorologia, Santa Maria, v.11, n.1, p.15-27, 2003.

DICKSON, A.; LEAF, A. L.; HOSNER, J. F. Quality appraisal of white spruce and white pine seedling stock in nurseries. Forestry Chronicle, Ottawa, v. 36, p. 10-13, 1960.

ESTAT. Sistema para análises estatísticas (v. 2.0). Jaboticabal: FCAV, UNESP, Departamento de Ciências Exatas, 1994.

FARIAS JUNIOR, J. A.; CUNHA, M. C. L.; FARIAS, S. G. G.; MENEZES JUNIOR, J. C. Crescimento inicial de mudas de turco sob diferentes tipos de recipientes e níveis de luminosidade. Revista Brasileira de Ciências Agrárias, Recife, v.2, n.3, p.228-232, 2007

KRAMER, P. J.; BOYER, J. S. Water relations of plants and soils. San Diego: Academic Press, 1995. $495 \mathrm{p}$.

MENDONCA, V.; ARRUDA, N. A. A.; SOUZA, H. A.; TEIXEIRA, G. A.; HAFLE, O. M.; RAMOS, J. D. Diferentes ambientes e osmocote ${ }^{\circledR}$ na produção de mudas de tamarindeiro (Tamarindus indica). Ciência e Agrotecnologia, Lavras, v. 32, n. 2, p. 391-397, 2008. 
MENDONÇA, V.; RAMOS, J. D.; CARRIJO, E. P.; PIO, R.; DANTAS, D. J.; GONTIJO, T. C. A. Diferentes ambientes e substratos na formação de mudas de mamoeiro 'Formosa'. Revista Científica Rural, Bagé, v. 10, n. 1, p.18-24, 2005.

MINAMI, K. Produção de mudas de alta qualidade em horticultura. São Paulo: Ed. Fundação Salim Sahad Maluf, 1995. 128p.

NEGREIROS, J. R. S.; ÁLVARES, V. S.; BRAGA, L. R.; BRUCKNER, C. H. Diferentes substratos na formação de mudas de maracujazeiro-amarelo. Revista Ceres, Viçosa, v. 51, n.294, p. 243-249, 2003.

PEREIRA, W. E.; SOUSA, G. G.; ALENCAR, M. L.; MENDOÇA, R. M. N.; SILVA, G. L. Crescimento de mudas de mamoeiro em substrato contendo caulim. Revista Verde de Agroecologia e Desenvolvimento Sustentável, Mossoró, v. 3, n. 1, p. 27-35, 2008.

RIBEIRO, A. C.; GUIMARÃES, P. T. G.; ALVAREZ, V. H. (Ed.). Recomendações para o uso de corretivos e fertilizantes em Mina Gerais: $5 \mathrm{a}$ aproximação. Viçosa: UFV, 1999. 359p.

SANO, S. M.; FONSECA, C. E. L. Estabelecimento de progênies de jatobá (Hymenaea spp) em plantios puros no Cerrado. Planaltina: Embrapa Cerrados, 2003. (Boletim de Pesquisa e Desenvolvimento, 110).
SANTOS, M. R.; NUNES, Y. R. F.; VELOSO, M. D. M.; RODRIGUES, P. M. S. Efeito do Vigor das Sementes no Crescimento inicial de mudas de jatobádo-cerrado (Hymenaea stigonocarpa - Fabaceae: Faboiedeae). Revista Brasileira de Biociências, Porto Alegre, v. 5, n.2, p. 261-263, 2007.

SILVA, A. J. N.; CABEDA, M. S. V.; CARVALHO, F. G. Matéria orgânica e propriedades físicas de um Argissolo Amarelo Coeso sob sistemas de manejo com cana-de-açúcar. Revista Brasileira de Engenharia Agrícola e Ambiental, Campina Grande, v. 10, n. 3, p. 579-585, 2006.

SILVA, M. R.; SILVA, M. A. A. P.; CHANG, Y. K. Uso de farinha de jatobá (Hymenaea stigonocarpa Mart.) em biscoitos tipo "cookie". Alimentos e Nutrição, Araraquara, v. 10, p. 7-22, 1999.

VIEIRA, E. A.; OLIVEIRA, A. M. R.; LAURA, V. A. Alocação de biomassa em plântulas de jatobá do cerrado (Hymenaea stigonocarpa Mart.) submetidas à competição. In: SIMPÓSIO NACIONAL SOBRE CERRADO, 9., SIMPÓSIO INTERNACIONAL DE SAVANAS TROPICAIS, 2., 2008, Brasília. Anais... Brasilia: EMBRAPA Cerrados, 2008.

ZANELLA, F.; SONCELA, R.; LIMA, A. L. S. Formação de mudas de maracujazeiro "amarelo" com níveis de sombreamento em Ji-Paraná/RO. Ciência e Agrotecnologia, Lavras, v. 30, n 5, p. 880-884, 2006. 